\title{
Properties of cellulosic material after cationization in
}

\section{different solvents}

\author{
Nora Odabas ${ }^{1}$, Hassan Amer ${ }^{1,2}$, Markus Bacher ${ }^{1}$, Ute Henniges ${ }^{1}$, Antje Potthast ${ }^{1}$, Thomas \\ Rosenau $^{l *}$ \\ ${ }^{1}$ Division of Chemistry of Renewable Resources, Department of Chemistry, University of \\ Natural Resources and Life Sciences Vienna, Konrad-Lorenz-Straße 24, 3430 Tulln, Austria \\ ${ }^{2}$ Department of Natural and Microbial Products Chemistry, National Research Centre, $33 \mathrm{Al}$ \\ Behous St., Dokki, Giza, Egypt
}

CONDUCTOMETRIC TITRATION DATA ANALYSIS

The degree of substitution was determined by conductometric titration. The conductivity values $(\sigma)$ were corrected for the increase in volume:

$$
\sigma_{\text {corrected }}=\sigma_{\text {measured }} \times \frac{\text { current volume }}{\text { initial volume }}
$$

In a homogeneous system, the point of change is a sharp knee in the conductivity curve. The system in the current study was heterogeneous, and there was rather an area of change. The DS 
was calculated using equivalence points derived from the intersection of the curves that are extended from the linear ranges before and after the area of change (see Figure S1).

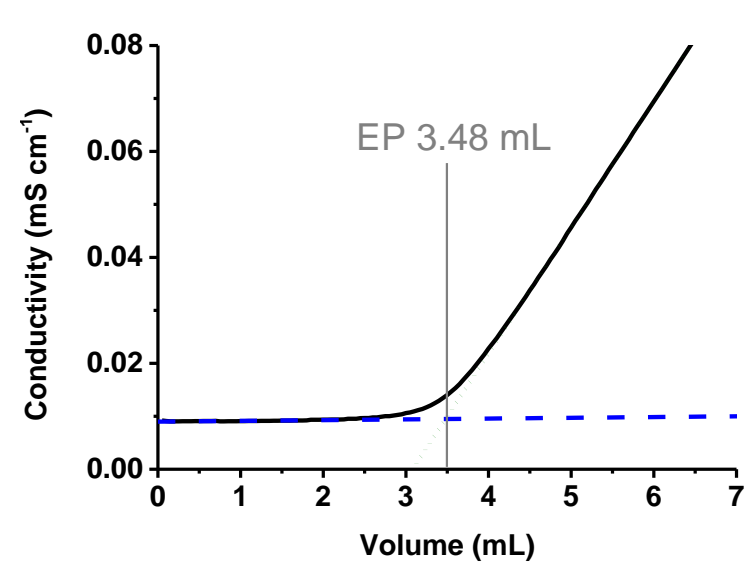

Figure S1. Example of a titration curve: Titration of about $50 \mathrm{mg}$ pulp IC; cryomilled. Conductivity (volume corrected) versus the volume of silver nitrate standard solution added (black solid line) plus the two straight lines before (blue, dashed) and after (green, dotted) that intersect in the equivalence point (grey vertical line).

The degree of substitution is defined as the number of functional groups per anhydroglucose unit. From the equivalence point $E P(\mathrm{~mL})$ and the dry mass of the titrated aliquot $(\mathrm{mg})$, the DS is calculated considering the concentration of the silver nitrate solution $c_{A g N O 3}\left(\mathrm{mmol} \mathrm{mL}^{-1}\right)$, the molar mass of an AGU $\left(M M_{A G U}=162 \mathrm{~g} \mathrm{~mol}^{-1}\right)$, and the molar mass of the functional group $\left(M M_{F G}=152 \mathrm{~g} \mathrm{~mol}^{-1}\right):$

$$
D S=\frac{E P \times c_{A g N O 3} \times M M_{A G U}}{\text { sample mass }-E P \times c_{A g N O 3} \times M M_{F G}}
$$




\section{CONTENT OF HIGHLY ORDERED AREAS IN CELLULOSE}

This study considers the term "crystallinity" figuratively and not in the strict sense of the

word. ${ }^{1}$ The three methods that were used to determine the content of highly ordered areas in cellulose were:

Total crystallinity

An index for total crystallinity is the ratio between the absorption band maxima at $1370 \mathrm{~cm}^{-1}$ and $2900 \mathrm{~cm}^{-1}$ (see Figure S2) as introduced by Nelson and $\mathrm{O}^{\prime}$ Connor ${ }^{2}$.

$$
C I_{A T R}=H_{1370} / H_{2900}
$$

The values of the absorption band maxima were determined as visualized in Figure S2: For $H_{2900}$, the local absorption minimum around $3000 \mathrm{~cm}^{-1}$ was subtracted as base from the absorption maximum around $2900 \mathrm{~cm}^{-1}$.

For $H_{1370}$, the base value to be subtracted from the absorption maximum around $1370 \mathrm{~cm}^{-1}$ was the linearly interpolated value from the local absorption minima around $1400 \mathrm{~cm}^{-1}$ and $1300 \mathrm{~cm}^{-1}$.

This ratio was calculated for every recorded spectrum. The results were averaged; the standard deviation of the results was recorded. 


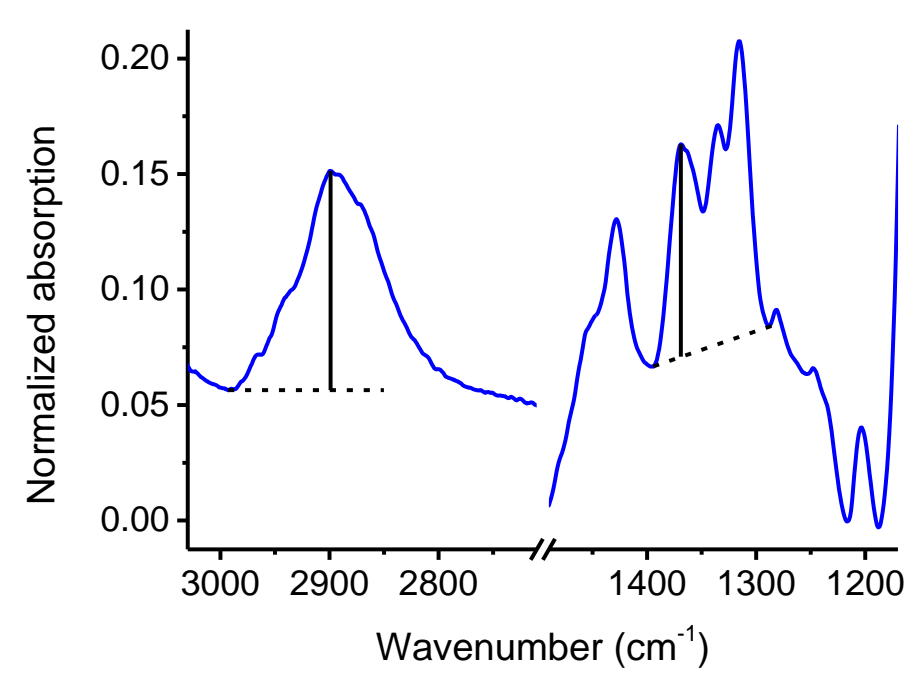

Figure S2. Data collection from ATR-FTIR spectra for determination of the "total crystallinity".

\section{Lateral order index}

"Lateral order index" is a name for the crystallinity index introduced by O'Connor et al. ${ }^{3}$ It is the ratio between the absorption band maxima at $1430 \mathrm{~cm}^{-1}$ (a measure for cellulose I) and $900 \mathrm{~cm}^{-1}$ (stronger for cellulose II and amorphous cellulose), see Figure S3.

$$
L O I=H_{1430} / H_{900}
$$

The values of the absorption band maxima were determined as visualized in Figure S3: For $H_{1430}$, the base value to be subtracted from the absorption maximum around $1430 \mathrm{~cm}^{-1}$ was the linearly interpolated value from the local absorption minima around $1500 \mathrm{~cm}^{-1}$ (should be zero) and $1400 \mathrm{~cm}^{-1}$.

For $H_{900}$, the base value to be subtracted from the absorption maximum around $900 \mathrm{~cm}^{-1}$ was the linearly interpolated value from the tangent to the curve around $850 \mathrm{~cm}^{-1}$ anchored to the local absorption minimum around $915 \mathrm{~cm}^{-1}$. 
This ratio was calculated for every recorded spectrum. The results were averaged; the standard deviation (SD) of the results was recorded. The value of this index was lower for material that had undergone blank treatment in isopropanol, see Figure S4.

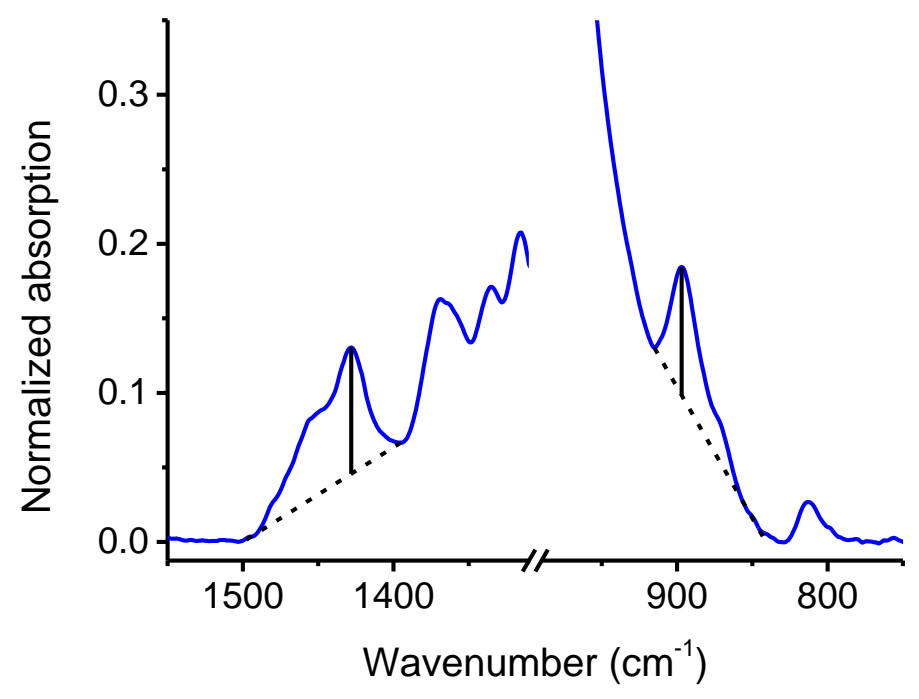

Figure S3. Data collection from ATR-FTIR spectra for determination of the "lateral order index".

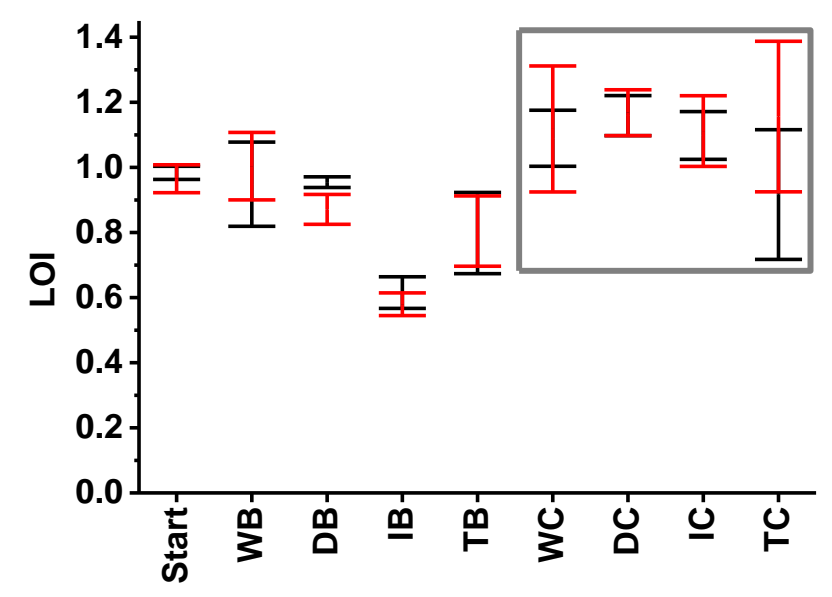

Figure S4. Lateral order index (LOI) for all samples. Values from two sets of experiments (black: first; red: second set), error bars: $2 \times$ standard deviation (SD). Values for cationized samples highlighted with grey rectangle. 
Crystallinity index derived from NIR spectra ${ }^{4}$

$$
C I_{N I R}=H_{4800} / H_{4800}+H_{5200}
$$

The values for $H_{4800}$ and $H_{5200}$ were taken from zero to the respective absorption maxima (see Figure S5), after rubberband baseline correction done by the instrument software as described in the experimental section. For cationized samples, the value was lower the higher the degree of substitution (see Figure S6), this probably reflects chemically increased hydrophilicity rather than a change in crystallinity. ${ }^{5}$

This ratio was calculated for every recorded spectrum. The results were averaged; the SD of the results was recorded.

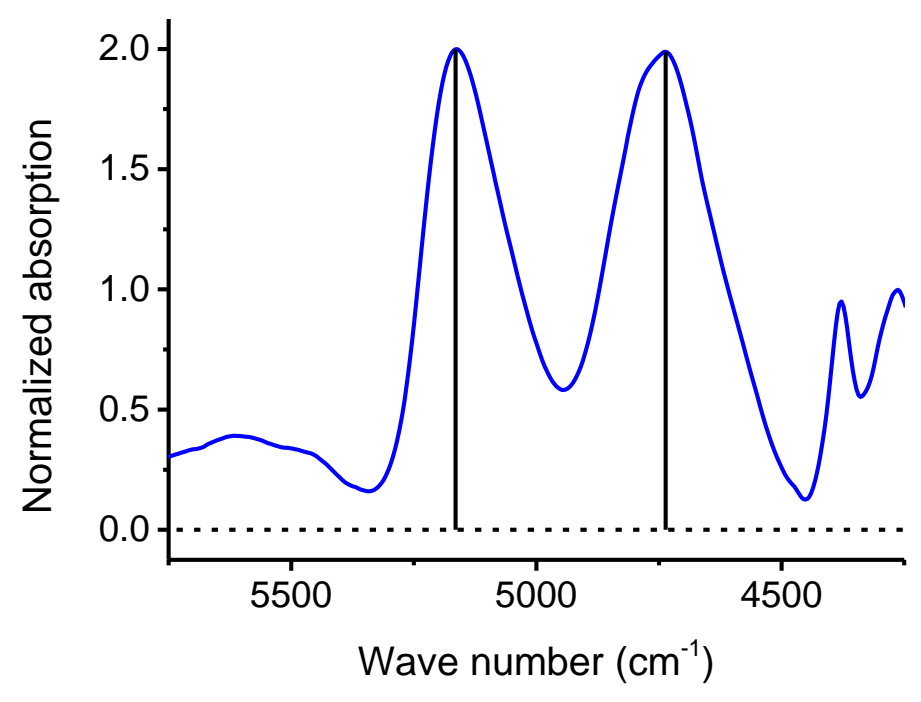

Figure S5. Data collection from NIR spectra for the determination of the respective crystallinity index. 


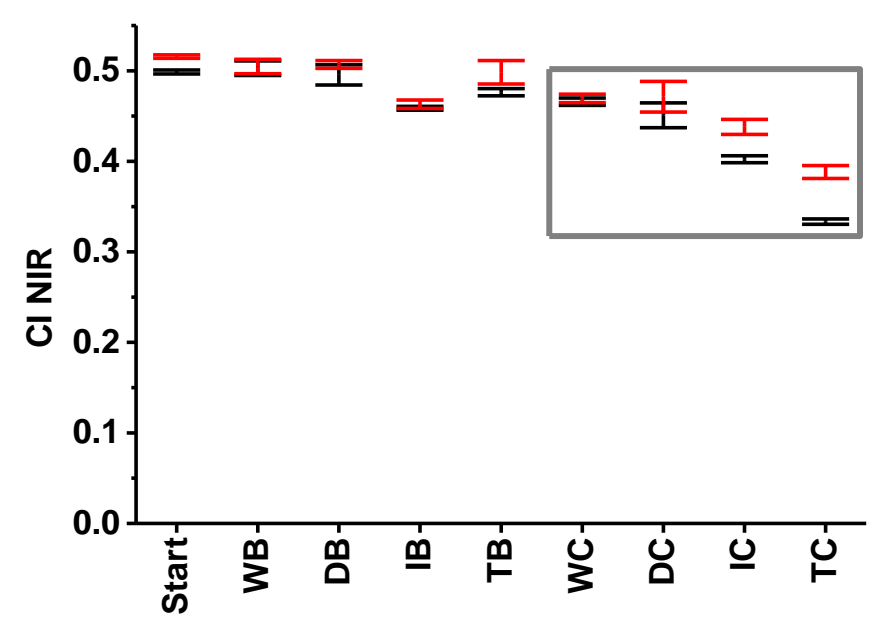

Figure S6. CI of the sample specimens determined from NIR. Values from two sets of experiments (black: first; red: second set), error bars: $2 \times$ SD. Values for cationized samples highlighted with grey rectangle.

\section{Solid-state NMR spectroscopy}

Solid-state CP/MAS ${ }^{13} \mathrm{C}$ NMR experiments were conducted as described by Siller et al. ${ }^{6}$ The spectra (see Figure S7 and Figure S8) were used to estimate the ratio of crystalline cellulose I to amorphous cellulose at C4. Fitting was hindered by too broad peaks although the materials were pre-swollen in water. Manual integration showed that the ratio of crystalline to amorphous material was $1: 0.94$ and $1: 0.92$ for the original pulp and the material blank treated with water (WB), respectively, with no cellulose II peaks being visible. The spectrum of DB was fairly similar. For IB, the ratio of crystalline to amorphous material was $1: 1.50$. The spectrum of TB showed more amorphous material than the original pulp, WB, and DB, but was still much closer to those than to IB. In the spectra of both IB and TB, the shoulder at $107.1 \mathrm{ppm}(\mathrm{C} 1)$ indicated the presence of some cellulose II, but the signal was too weak to allow for quantification. 


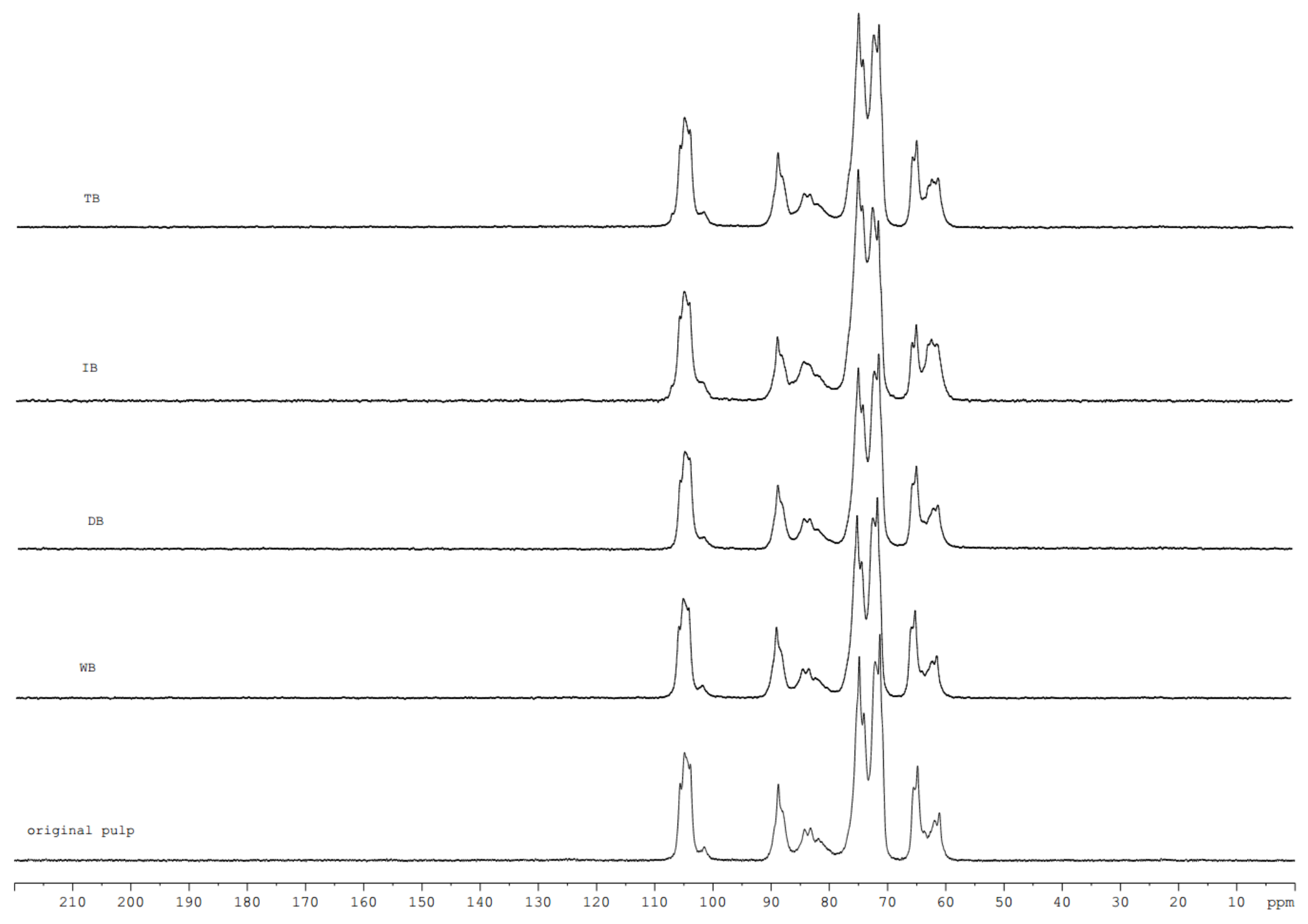

Figure S7. Solid-state ${ }^{13} \mathrm{C}$ NMR spectra of (from bottom to top) original pulp, WB, DB, IB, and TB; overview. 


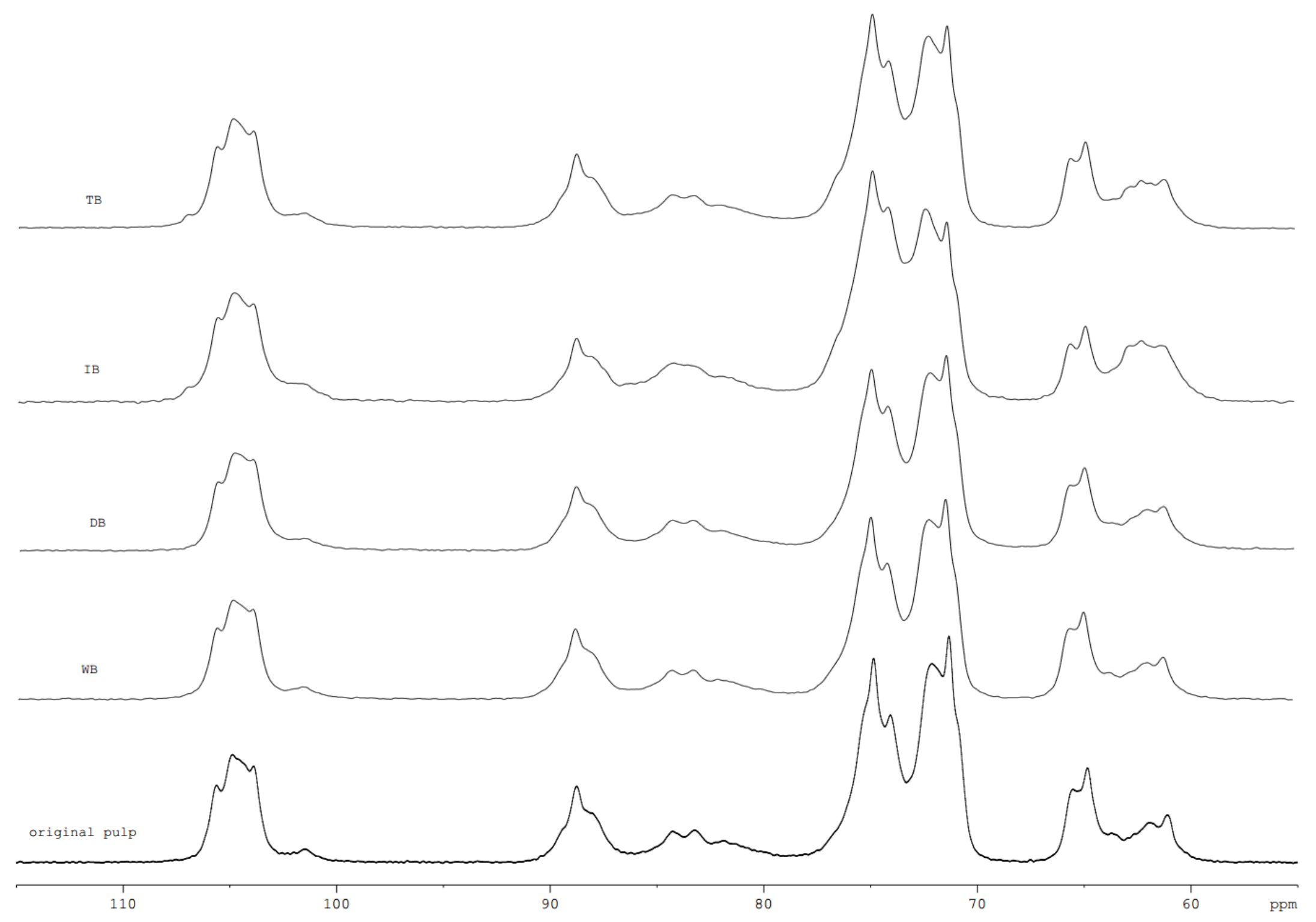

Figure S8. Solid-state ${ }^{13}$ C NMR spectra of (from bottom to top) original pulp, WB, DB, IB, and TB; section of relevant chemical shift magnified. 


\section{REFERENCES}

(1) Newman, R. H.; Hill, S. J.; Harris, P. J. Wide-Angle X-Ray Scattering and Solid-State Nuclear Magnetic Resonance Data Combined to Test Models for Cellulose Microfibrils in Mung Bean Cell Walls. Plant Physiol. 2013, 163, 1558-1567.

(2) Nelson, M. L.; O'Connor, R. T. Relation of certain infrared bands to cellulose crystallinity and crystal lattice type. Part II. A new infrared ratio for estimation of crystallinity in celluloses I and II. J. Appl. Polym. Sci. 1964, 8, 1325-1341.

(3) O'Connor, R. T.; DuPré, E. F.; Mitcham, D. Applications of Infrared Absorption Spectroscopy to Investigations of Cotton and Modified Cottons: Part I: Physical and Crystalline Modifications and Oxidation. Text. Res. J. 1958, 28, 382-392.

(4) Basch, A.; Wasserman, T.; Lewin, M. Near-infrared spectrum of cellulose: A new method for obtaining crystallinity ratios. J. Polym. Sci., Polym. Chem. Ed. 1974, 12, 1143-1150.

(5) Schwanninger, M.; Rodrigues, J.; Fackler, K. A review of band assignments in near infrared spectra of wood and wood components. J. Near Infrared Spectrosc. 2011, 19, 287-308.

(6) Siller, M.; Amer, H.; Bacher, M.; Roggenstein, W.; Rosenau, T.; Potthast, A. Effects of periodate oxidation on cellulose polymorphs. Cellulose 2015, 1-17. 